\title{
Aumento de solicitações de dímero-D na primeira onda de COVID-19 em um hospital universitário da região metropolitana do Estado do Rio de Janeiro
}

Increase in requests for D-dimer in the first wave of COVID-19 in a university hospital in the metropolitan region of the State of Rio de Janeiro

Aumento de solicitudes de dímero D en la primera ola de COVID-19 en un hospital universitario de la región metropolitana del estado de Río de Janeiro

Paulo Sérgio de Abreu Junior ORCID: https://orcid.org/0000-0002-7989-3504 Universidade Federal Fluminense, Brasil E-mail: paulo_abreu@id.uff.br

Suelen Brito Nascimento ORCID: https://orcid.org/0000-0002-6566-8402 Universidade Federal Fluminense, Brasil E-mail: suelenbrito@id.uff.br

Sara Nascimento

ORCID: https://orcid.org/0000-0002-3979-0244 Universidade Federal Fluminense, Brasil

E-mail: sara_nascimento@id.uff.br

Gabriel Dourado

ORCID: https://orcid.org/0000-0002-4837-2589 Universidade Federal Fluminense, Brasil E-mail: gabrieldourado@id.uff.br

Marianna Gomes

ORCID: https://orcid.org/0000-0001-5382-6605 Universidade Federal Fluminense, Brasil

E-mail: marianna_gomes@id.uff.br

Jenifer Pereira Vitoriano

ORCID: https://orcid.org/0000-0002-6000-4316 Universidade Federal Fluminense, Brasil E-mail: jeniferpv@id.uff.br

Iasmin Cardoso Fonseca

ORCID: https://orcid.org/0000-0002-2840-6526 Universidade Federal Fluminense, Brasil E-mail: iasminicf@gmail.com

Hye Chung Kang

ORCID: https://orcid.org/0000-0002-5139-122X Universidade Federal Fluminense, Brasil E-mail: hyekang@id.uff.br

\begin{abstract}
Resumo
Introdução: O dímero-D é um produto de degradação da fibrina, sendo marcador indireto da fibrinólise. Se um indivíduo infectado por SARS-COV 2 evolui com agravamento, o resultado igual ou superior a quatro vezes o valor de referência deste marcador no momento da internação é considerado um preditor de mortalidade. O exame ganhou maior importância desde o início da pandemia, devido às manifestações trombóticas decorrentes da infecção. Objetivo: Avaliar a variação no quantitativo de solicitações médicas para dosagem do dímero-D em um dado Hospital Universitário e seu perfil na primeira onda da pandemia da COVID-19. Metodologia: Foi realizado um estudo observacional transversal, onde foram quantificados e analisados exames de pacientes com perfil de COVID-19 identificados no Laboratório de Urgências de um Hospital Universitário, foram consideradas solicitações médicas para dosagem de dímero-D no período de 03/01/2020 a 03/07/2020. Resultados: No primeiro semestre de 2020 observamos 1017 solicitações. Comparando o $1^{\circ}$ trimestre com o $2^{\circ}$ trimestre do mesmo ano verificou-se um aumento abrupto de $1274 \%$ no total de solicitações de um trimestre para o outro, uma vez que no $1^{\circ}$ foram detectadas apenas 69 solicitações com mediana $2231 \mathrm{ng} / \mathrm{mL}$ (intervalo interquartil 175-15605), já no $2^{\circ}$ trimestre esse número saltou para um total de 948 solicitações com mediana $2020 \mathrm{ng} / \mathrm{mL}$ (intervalo interquartil 99-97026). Conclusão: Embora o dímero-D não tenha sua especificidade elevada enquanto ferramenta de diagnóstico, tem sido muito útil como preditor de gravidade para COVID-19. A despeito de o enorme impacto causado pelo aumento substancial na demanda de solicitações de dímero-D neste Hospital Universitário não ter provocado solução de continuidade na prestação dos serviços, maiores estudos em gestão de riscos e de
\end{abstract}


calamidades seriam oportunos, a fim de se aprimorar o fluxo resolutivo para situações que, infelizmente, possam vir a ocorrer.

Palavras-chave: Fibrinólise; Dímero; Pandemia.

\begin{abstract}
Introduction: D-dimer is a fibrin degradation product and is an indirect marker of fibrinolysis. If an individual infected with SARS-COV 2 evolves with aggravation, a result equal to or greater than four times the reference value of this marker at the time of admission is considered a predictor of mortality. The most important exam since the beginning of the pandemic, due to the thrombotic manifestations resulting from the infection. Objective: To evaluate the variation in the number of medical requests for D-dimer dosage at a given University Hospital and its profile in the first wave of the COVID-19 pandemic. Methodology: This is a cross-sectional observational study, where the results of patients with a COVID-19 profile, identified in the Emergency Laboratory of a University Hospital, were quantified and medical causes for D-dimer dosages in the period from 03/01/2020 to 07/03/2020. Results: In the first half of 2020, we observed 1017 requests. Comparing the 1 st quarter with the 2 nd quarter of the same year, there was an abrupt increase of $1274 \%$, no total occurrences from one quarter to the other, since no 1st quarter, only 69 requests were detected with a median 2231 $\mathrm{ng} / \mathrm{mL}$ ( interquartile range 175-15605), no longer 2nd quarter this number jumped to a total of 948 requests with a median $2020 \mathrm{ng} / \mathrm{mL}$ (interquartile range 99-97026). Conclusion: Although D-dimer does not have high specificity as a diagnostic tool, it has been very useful as a severity predictor for COVID-19. Despite the enormous impact generated by the substantial increase in demand for D-dimer demand in this University Hospital, have provoked a solution of continuity in the provision of services, further studies in risk and disaster management would be opportune, in order to improve the resolving flow for situations that, unfortunately, may occur.
\end{abstract}

Keywords: Fibrinolysis; Dimer; Pandemic.

\title{
Resumen
}

Introducción: El dímero D es un producto de degradación de la fibrina y es un marcador indirecto de la fibrinólisis. Si un individuo infectado con SARS-COV 2 evoluciona con agravación, un resultado igual o mayor que cuatro veces el valor de referencia de este marcador en el momento del ingreso se considera un predictor de mortalidad. El examen más importante desde el inicio de la pandemia, por las manifestaciones trombóticas derivadas de la infección. Objetivo: Evaluar la variación en el número de solicitudes médicas de dosis de dímero D en un Hospital Universitario dado y su perfil en la primera ola de la pandemia de COVID-19. Metodología: Se trata de un estudio observacional transversal, donde se cuantificaron los resultados de los pacientes con perfil COVID-19, identificados en el Laboratorio de Urgencias de un Hospital Universitario y las causas médicas de las dosis de dímero D en el período del 03/01/2020 al 07/03/2020. Resultados: En el primer semestre de 2020, observamos 1017 solicitudes. Comparando el 1er trimestre con el 2 do trimestre del mismo año, hubo un incremento abrupto de $1274 \%$, no hubo ocurrencias totales de un trimestre al otro, ya que no se detectó 1er trimestre, solo se detectaron 69 solicitudes con una mediana de $2231 \mathrm{ng} / \mathrm{mL}$ (rango intercuartílico 175-15605), ya no en el segundo trimestre, este número saltó a un total de 948 solicitudes con una mediana de $2020 \mathrm{ng} / \mathrm{ml}$ (rango intercuartílico 99-97026). Conclusión: Aunque el dímero D no tiene una alta especificidad como herramienta diagnóstica, ha sido muy útil como predictor de gravedad para COVID-19. A pesar del enorme impacto generado por el aumento sustancial de la demanda de dímero D en este Hospital Universitario, se han propiciado una solución de continuidad en la prestación de servicios, sería oportuno realizar más estudios en gestión de riesgos y desastres, con el fin de mejorar el flujo de resolución de situaciones que, lamentablemente, puedan ocurrir.

Palabras clave: Fibrinólisis; Dímero; Pandemia.

\section{Introdução}

A COVID-19 é uma doença infecciosa que afeta principalmente o sistema respiratório, e tem como agente etiológico o vírus da síndrome respiratória aguda grave 2 (SARS-CoV-2).(Connors \& Levy, 2020; Cunningham et al., 2020; Hanff et al., 2020; Higuera-de-la-Tijera et al., 2021; Li et al., 2020; Rostami \& Mansouritorghabeh, 2020; Zhang et al., 2020) Desde o final de 2019 começou a se alastrar pelo mundo, sendo declarada pandemia pela OMS em 11 de março de 2020.(BRASIL, 2020) Desde então, a política de saúde pública no Brasil e no mundo, precisa lidar com inúmeros desafios, sobretudo no aspecto da gestão de riscos e de calamidades, fator indispensável na contenção de maiores desdobramentos pelo atual momento pandêmico.(Quacken et al., 2021; Velicu et al., 2021; WHO, 2010)

O mundo ainda está tentando compreender a fisiopatologia da doença e seus diversos biomarcadores de gravidade. Dentre os quais, podemos citar o dímero-D, cujo resultado igual ou superior a quatro vezes o valor de referência no momento da internação é considerado um preditor de mortalidade.(Debuc \& Smadja, 2021; Silla, L. R. M., 2005; Hanff et al., 2020; Harper et al., 2007) Em uma revisão sistemática e meta-análise realizada por Gabbai-Armelin, P. R. et al. em 2021, ficou evidente que 
pacientes gravemente acometidos por COVID-19 tendem a apresentar níveis séricos pronunciadamente mais elevados de dímeroD em comparação com pacientes menos graves, acometidos pela mesma doença.(Gabbai-Armelin et al., 2021)

Os níveis de dímero-D estão intimamente ligados à atividade fibrinolítica, uma vez que este analito é um dos produtos de degradação da fibrina (PDF), após ativação da coagulação e consequente formação de um trombo. Os PDF surgem a partir de 3 reações: conversão de fibrinogênio em fibrina pela trombina, reticulação de fibrina pelo fator XIII ativado e degradação da fibrina pela plasmina, originando produtos de diferentes pesos moleculares.(Johnson et al., 2019; Weitz et al., 2017) Destes, o menor e melhor analito para expressar a atividade trombolítica é o dímero - D. Por possuir uma meia-vida relativamente curta de aproximadamente 8 horas, esse produto de degradação da fibrina é muito utilizado para avaliar a ativação do processo de formação do trombo e sua fibrinólise decorrente.(Silla, L. R. M., 2005; Johnson et al., 2019; Moreno et al., 2021; Weitz et al., 2017)

Em situações normais, baixos níveis de dímero-D podem ser detectados na circulação sanguínea de indivíduos saudáveis, porém, seus níveis podem aumentar significativamente em pacientes com coagulação intravascular disseminada (CIVD), embolia pulmonar (EP), trombose venosa profunda (TVP), infarto do miocárdio, câncer, complicações na gravidez, sepse, dentre outros fatores.(Moreno et al., 2021; Rostami \& Mansouritorghabeh, 2020; Wu et al., 2020; Zhang et al., 2020) Além disso, o dímero-D pode sofrer grande variação, particularmente, em decorrência da idade(Harper et al., 2007) e, nesses casos, não se sustenta como parâmetro ideal, se observado isoladamente, na distinção entre os pacientes com trombose daqueles pacientes sem trombose caso apresentem idade superior a 60 anos, hospitalização por um período maior que 03 dias ou, ainda, apresentarem elevados níveis de proteína C reativa.(Harper et al., 2007; Moreno et al., 2021; Rostami \& Mansouritorghabeh, 2020)

Apesar de suas possíveis variações em decorrência dos fatores supramencionados, atualmente, reconhece-se o dímeroD como o melhor marcador para trombose e fibrinólise fisiológica, e valores inferiores a $500 \mathrm{ng} / \mathrm{mL}$ ainda contribuem sobremaneira para a exclusão diagnóstica de eventos trombóticos ou tromboembólicos na rotina clínica.(Silla, L. R. M., 2005; Harper et al., 2007; Johnson et al., 2019; Rostami \& Mansouritorghabeh, 2020)

Assim, em virtude de sua estreita correlação com eventos trombóticos e seus consequentes agravos em pacientes acometidos por COVID-19, o dímero-D tem sido largamente utilizado no auxílio diagnóstico e prognóstico dessa doença. (Harper et al., 2007; Li et al., 2020; Moreno et al., 2021; Rostami \& Mansouritorghabeh, 2020; Zhang et al., 2020) E, dessa maneira, este trabalho visa avaliar a variação no quantitativo de solicitações médicas para dosagem do dímero-D em um hospital universitário da região metropolitana do Estado do Rio de Janeiro e seu perfil na primeira onda da pandemia de COVID-19, procurando discutir a utilização deste analito, bem como propor algumas medidas que poderiam amenizar os impactos do consumo demasiado em uma semelhante situação posterior.

\section{Metodologia}

Este trabalho obteve aprovação do Comitê de Ética e Pesquisa de Nova Friburgo-RJ (parecer consubstanciado n ${ }^{\circ}$ 4.238.493), e inicialmente foi realizado um estudo comparativo da média semestral do número de solicitações para dosagem do dímero-D em 2019 com o número global de solicitações no $1^{\circ}$ semestre de 2020, a fim de se obter uma comparação entre os períodos pré e intra-pandêmicos. Posteriomente, foi realizado um estudo observacional transversal (Pereira et al., 2018) considerando solicitações médicas para dosagem de dímero-D no período de 03/01/2020 a 03/07/2020 em um Hospital Universitário na região metropolitana do Estado do Rio de Janeiro. Os dados foram levantados através de pesquisa qualitativa nos prontuários dos pacientes internados com solicitações de dímero - $\mathrm{D}$, utilizando-se auxílio do sistema informatizado do Serviço de Patologia Clínica. Estes dados foram tabulados em uma planilha Excel, tendo sido realizada uma análise descritiva e os valores expressos em média, mediana e desvio padrão conforme cada caso. Para as pesquisas qualitativas e quantitativas das 
fontes de informação foram confeccionados os gráficos no Programa GraphPad Prism, e utilizadas as bases de dados PUBMED, LILACS, SCIELO e MEDLINE. As variáveis numéricas foram distribuídas em média e foram utilizados o teste estatístico de Kruskal-Wallis e teste de comparação múltipla de Dunn.

\section{Resultados e Discussão}

\section{$\bullet 2019$ vs 2020}

Inicialmente foi realizado um estudo comparativo da média semestral do $\mathrm{n}^{\circ}$ de solicitações para dosagem do dímero-D no ano de 2019 com o número global de solicitações no $1^{\circ}$ semestre de 2020, a fim de se obter uma comparação entre os períodos pré e intra-pandêmicos, correlacionando-se um período declaradamente anterior à pandemia por COVID-19 com outro já inserido nesse contexto.

Desta maneira, obtiveram-se 360 solicitações em todo o ano de 2019, contrastando-se com 1017 solicitações apenas no primeiro semestre de 2020 (Figura 1), um aumento de 182,50\% entre esses dois períodos. A fim de se facilitar a comparação do $\mathrm{n}^{\mathrm{o}}$ de solicitações entre os períodos correspondentes a ambos os semestres nos dois anos, foram correlacionados o $\mathrm{n}^{\mathrm{o}}$ de solicitações do $1^{\circ}$ semestre de 2019 (185) com o n ${ }^{\circ}$ de solicitações obtidas no $1^{\circ}$ semestre de 2020 (1017).

Figura 1: Correlação entre o n ${ }^{\circ}$ de solicitações do $1^{\circ}$ semestre de 2019 com o n ${ }^{\circ}$ de solicitações no $1^{\circ}$ semestre de 2020.

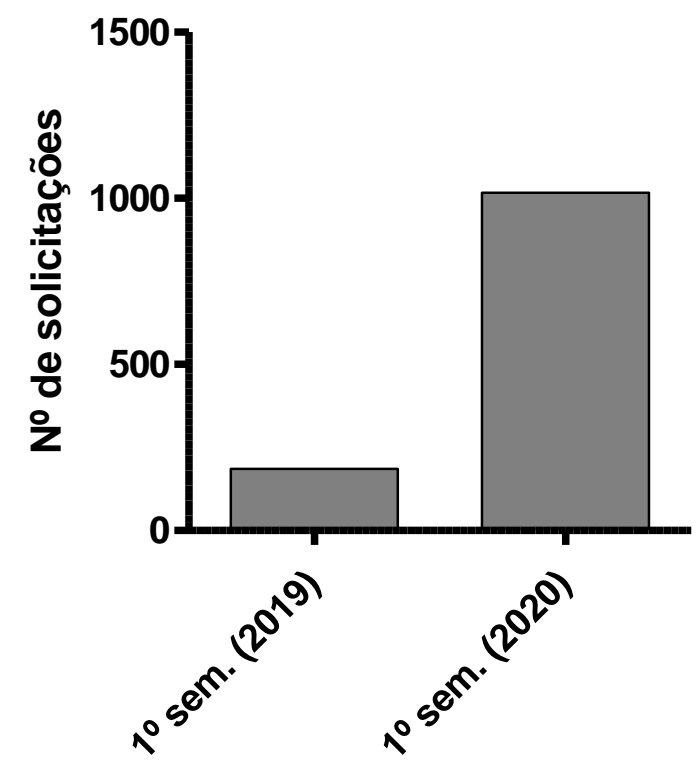

Fonte: Dados de prontuários dos pacientes.

Com esse aumento extraordinário nas análises envolvendo o dímero-D, houve rápida superação da previsão de demanda deste Hospital Universitário, sendo relatado pela Chefia do Serviço de Patologia Clínica que, em poucos meses, houve esgotamento do saldo para aquisição destes insumos no contrato de fornecimento anual de reagentes, o que levou a administração a realizar diversos procedimentos emergenciais, como empréstimo adquirido de outras unidades, compra emergencial de reagentes para dosagem de dímero-D, bem como aumento da estimativa deste analito em pregão posterior. Além disso, houve empréstimo de equipamento e reagentes como forma de backup, para que a demanda fosse suprida a contento, visando-se coibir ou minimizar ao máximo qualquer solução de continuidade na prestação do serviço. 
Cabe salientar, ainda, que os reagentes para dosagem de dímero - D fornecidos à instituição em estudo são importados, e com o aumento da demanda por esse reagente somado à diminuição do tráfego aéreo por ocasião das medidas de contenção durante pandemia, houve deficiência na manutenção dos estoques por diversas empresas fornecedoras de tal insumo, contribuindo ainda mais para o seu esgotamento por parte da empresa licitante neste hospital.

Contudo a realização das dosagens de dímero - D foi garantida a contento com a utilização de um outro equipamento, porém ocasionando a mudança de metodologia empregada, que antes era realizada por imunoturbidimetria, utilizando o equipamento STA-R EVOLUTION e, num dado intervalo do período avaliado, passou a se dar por imunofluorescência, utilizando o equipamento TRIAGE, retornando posteriormente à utilização do primeiro. Os valores de referência para o dímero - D não foram alterados, porém tal medida levou a alguns impactos na rotina, dentre os quais podemos citar: treinamento ostensivo de toda equipe envolvida nas análises; reformulação do laudo, contemplando a nova metodologia; utilização de amostra de sangue total, em vez de soro; aumento da carga de trabalho da equipe, seja no âmbito administrativo, a fim de viabilizar novas aquisições que suprissem o referido esgotamento, seja no âmbito técnico-operacional, com uma metodologia que exigia maior tempo e maior quantidade de operações manuais para sua execução.

Dos impactos provocados, provavelmente o de maior influência teria sido o aumento no tempo de liberação dos laudos em decorrência de a nova metodologia limitar-se à análise de uma amostra por vez. Este fator, sem dúvida, teve ainda maior influência quando da necessidade de execução de diversas amostras recebidas em intervalo curto de tempo.

Ademais, os amplos e generalizados impactos ocasionados pela pandemia notoriamente tiveram desdobramentos sem precedentes nas políticas de saúde públicas, não apenas pelo Brasil mas também mundo afora.(Chen et al., 2020; Velicu et al., 2021) O esgotamento da oferta desses reagentes ocorreu de forma difusa, não apenas em um, como em outros hospitais, o que eleva as considerações de atendimento das demandas gerenciais a um prisma multifatorial e complexo.

No entanto, a despeito do caráter multifatorial (Chen et al., 2020; Velicu et al., 2021) que teria culminado em interrupção do fornecimento de reagentes licitados, obrigando a administração pública a ações sanadoras a fim de garantir a continuidade na prestação dos serviços, a necessidade de importação de insumos somada à diminuição do tráfego aéreo parece ter tido maior peso de contribuição no desabastecimento momentâneo destes insumos no laboratório em estudo, motivo pelo qual uma revisão dos protocolos de ações em situações de calamidade seriam oportunos como medida de prevenção no caso de uma próxima eventualidade.

\section{- 2020 (períodos pré e intrapandêmico)}

A fim de se obter uma melhor análise do impacto causado pela pandemia no número de solicitações de dímero-D neste hospital, foram compilados os dados referentes às solicitações no período de 03/01/2020 a 03/07/2020, compreendendo os 03 meses antecedentes e os 03 meses posteriores à declaração da pandemia pela OMS, em 11 de março de 2020 (WHO, 2010), tendo sido identificadas 1017 solicitações no $1^{\circ}$ semestre de 2020, das quais 963 teriam resultados válidos e analisáveis, sendo excluídas 54 solicitações com resultados incomputáveis, perfazendo um total de 5,31\% de resultados inválidos e 94,69\% de resultados válidos do total de solicitações observadas.

O que se verificou foi um aumento abrupto de $1274 \%$ no total de solicitações de um trimestre para o outro, uma vez que no $1^{\circ}$ trimestre foram obtidas apenas 69 solicitações, já no $2^{\circ}$ trimestre esse número saltou para um total de 948 solicitações para dosagem de dímero - D (Figura 2-A). Esse expressivo aumento fica ainda mais evidenciado quando visto mês a mês (Figura 2-B) 
Figura 2-A: $n^{\circ}$ de solicitações obtidas no $1^{\circ}$ trimestre e $n^{\circ}$ de solicitações obtidas no $2^{\circ}$ trimestre de 2020; 2-B: $n^{\circ}$ de solicitações obtidas mês a mês no $1^{\circ}$ semestre de 2020 .

A)

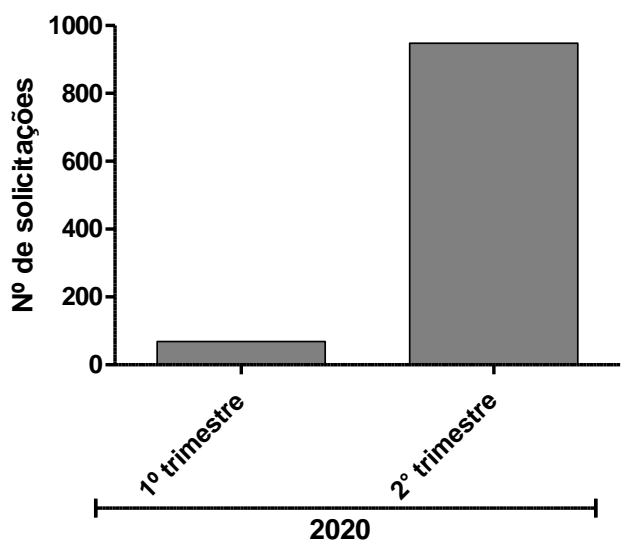

B)

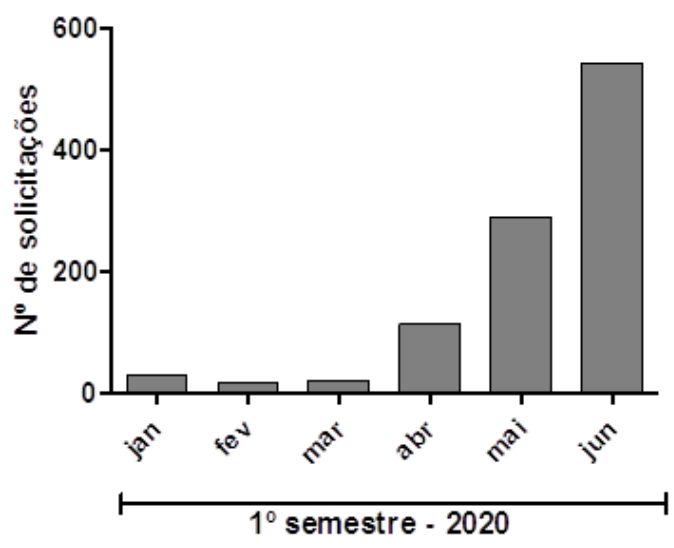

Fonte: dados de prontuários dos pacientes.

O aumento pronunciado nas solicitações de dímero - D revela o impacto provocado pelo período pandêmico e, embora inespecífico para o diagnóstico da doença em si, o analito teve e ainda tem elevada importância como preditor de mortalidade em pacientes que chegam à unidade de saúde com suspeita ou sabidamente acometidos por COVID-19.(Li et al., 2020; Zhang et al., 2020) Existem protocolos de monitoramento dos valores de dímero-D em intervalos de 24 a 48 horas desde a internação do paciente, no intuito de melhor avaliar seu prognóstico.(BRASIL, 2020) O maior número de solicitações ficou entre os pacientes do sexo masculino, com um total de $544(53,49 \%)$ para homens. Para o sexo feminino foram quantificadas 425 solicitações $(46,51 \%)$.

Essa diferença entre os sexos é mais expressiva no trimestre pós declaração da pandemia, como visto na Figura 3, em que se correlacionou o número de solicitações de dímero - D categorizados por $1^{\circ}$ e $2^{\circ}$ trimestres de 2020 , de acordo com sexo dos pacientes. 
Figura 3: $n^{\circ}$ de solicitações de dímero - D categorizados por $1^{\circ}$ e $2^{\circ}$ trimestres de 2020 , de acordo com sexo dos pacientes.

\section{0}

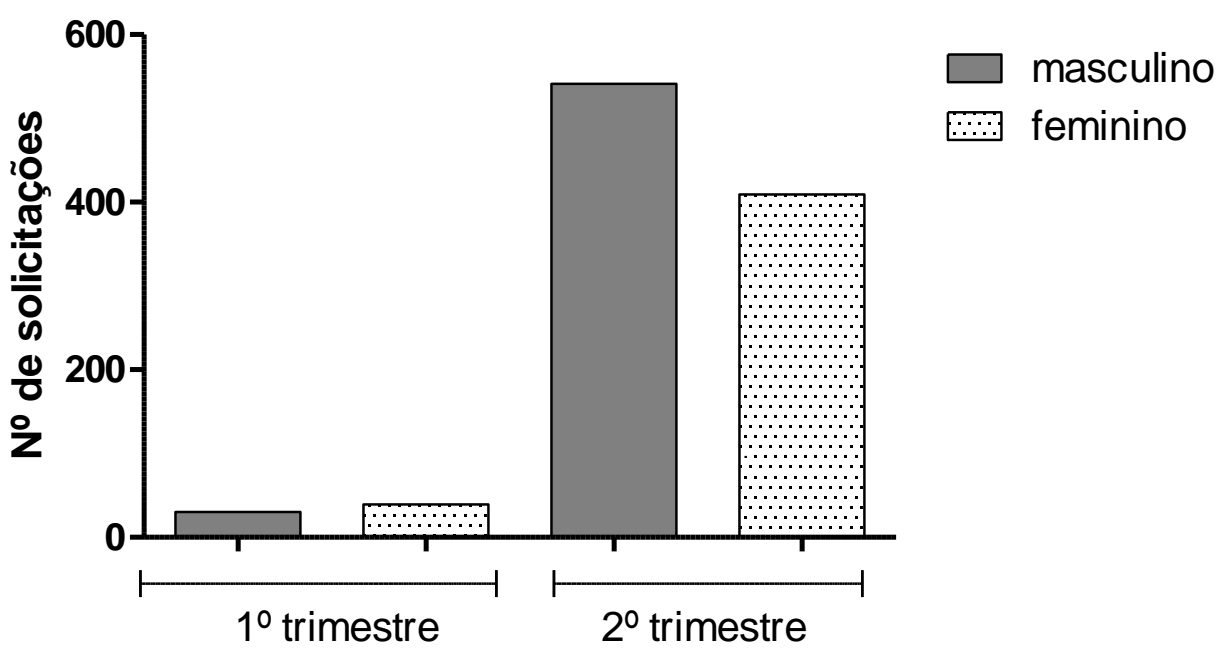

Fonte: Dados de prontuários dos pacientes.

Talvez o $\mathrm{n}^{\circ}$ de solicitações ligeiramente aumentado para homens (544; sendo 53,49\% ) em comparação às mulheres (425, sendo 46,51\%) seja influenciado por estudos recentes que apontam que, embora homens e mulheres tenham semelhante suscetibilidade para contrair o vírus, os homens parecem ter maior propensão a desenvolver formas graves da doença e apresentam taxa de mortalidade superior à das mulheres também acometidas pela COVID-19 (Chen et al., 2020; Jin et al., 2020).

Com relação à média de idade dos pacientes nas solicitações, esta foi de 55 anos para o $1^{\circ}$ trimestre e $63( \pm 5,66)$ anos para o $2^{\circ}$, representando um aumento de $14,54 \%$, predizendo um ligeiro aumento na média de idade dos pacientes do período pós pandemia declarada, se contrastado com a média de idade dos pacientes no período pré declaração da pandemia pela OMS.

Não se pôde inferir, neste estudo, se o aumento das solicitações visando ao rastreio do dímero-D em pacientes de idade aumentada teria ocorrido devido à preocupação dos médicos prescritores com o fato de que estes pacientes (acima de 60 anos) têm maior predisposição a desenvolver as formas graves da doença(Harper et al., 2007; Rahi et al., 2021; Wu et al., 2020), embora nesta idade os valores de dímero-D sofram grande interferência e não se prestem a reproduzir com fidedignidade uma correlação com eventos trombóticos(Harper et al., 2007; Li et al., 2020; Rostami \& Mansouritorghabeh, 2020), ou se estes pacientes foram os mais acometidos ao ponto de procurar atendimento médico.

Já a média dos valores de dímero-D dosados de janeiro a junho podem ser vistas na Figura 4. A média mínima foi obtida no mês de abril, com dosagem de 2045 ng/mL, e a média máxima no mês seguinte, em maio, sendo de 3609 ng/mL. Nesta figura, a média dos valores de dímero - D (ng/mL) foi calculada a cada mês do $1^{\circ}$ semestre de 2020 . * Dados obtidos com diferença significativa por estatística de Kruskal-Wallis e teste de comparação múltipla de Dunn $(\mathrm{p}<0,05)$ referente aos meses de abril/maio e abril/junho. 
Figura 4: média dos valores de dímero-D observados de janeiro a junho de 2020.

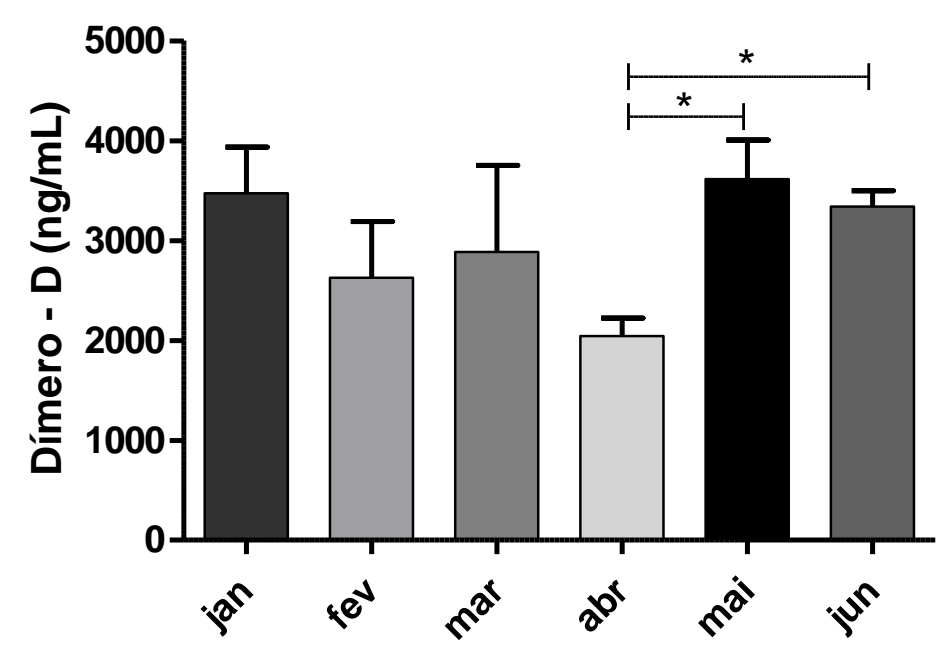

Fonte: Dados de prontuários dos pacientes.

Apesar de o dímero - D estar caracteristicamente aumentado em pacientes acometidos por COVID-19, a média desses valores no $2^{\circ}$ trimestre de $2020(2029$ ng/mL), pós pandemia declarada, expressou-se com uma redução de 51,06\% em relação ao trimestre anterior $(3065 \mathrm{ng} / \mathrm{mL})$.

O resultado dessa diminuição na média dos valores de dímero-D durante o período pandêmico deve ser contextualizado sob a possibilidade de o primeiro período tratar-se de possíveis solicitações mais direcionadas a pacientes sabidamente com eventos trombóticos, em contraste com as solicitações menos direcionadas e mais preventivas para as formas graves de COVID19 no segundo período, talvez abarcando um maior percentual de pacientes sem histórico de eventos trombóticos nesta pesquisa.

Na Figura 5, tem-se o número de casos das dosagens de dímero - D categorizadas por intervalos de resultados, em ng/mL e prevalência dos sexos dos pacientes, compreendidos no $1^{\circ}$ semestre de 2020 . Podemos observar que o menor número de casos foi referente ao intervalo "acima de $10.000 \mathrm{ng} / \mathrm{mL}$ ", com apenas 51 casos, enquanto o maior número de casos foi o do intervalo de 2000 a $5000 \mathrm{ng} / \mathrm{mL}$, com 298 casos. 
Figura 5: $\mathrm{n}^{\mathrm{o}}$ de casos das dosagens de dímero - D categorizadas por intervalos de resultados, em ng/mL e prevalência dos sexos dos pacientes, compreendidos no $1^{\circ}$ semestre de 2020.

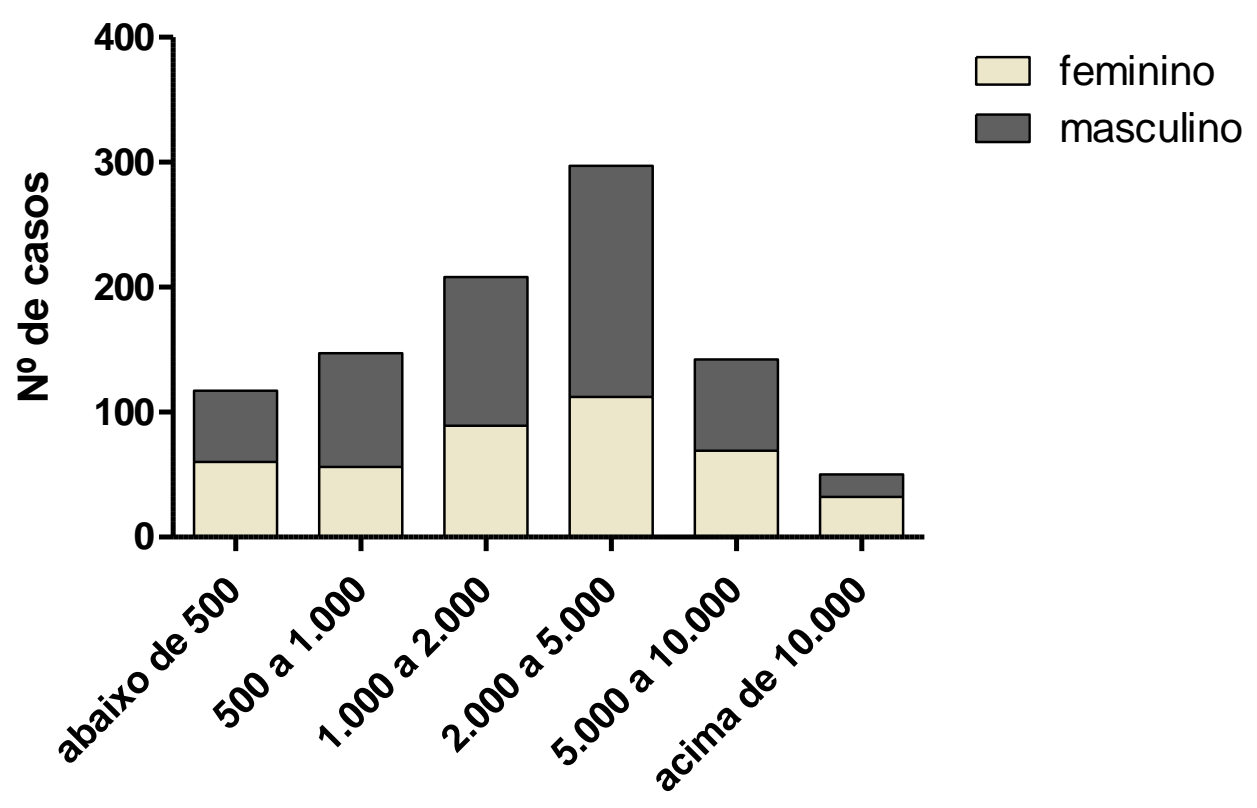

Fonte: dados de prontuários dos pacientes.

Dos valores de dímero-D acima de $10.000 \mathrm{ng} / \mathrm{mL}$, os três maiores foram plotados na tabela abaixo (Legenda: F feminino; CTI - Centro de Tratamento Intensivo; CD - Centro de Diálise; Ca - câncer; HAS - hipertensão arterial sistêmica; DPOC - doença pulmonar obstrutiva crônica; e DRC - doença renal crônica):

Tabela 1: Observações a respeito dos 03 maiores valores de dímero - D.

\begin{tabular}{cccc}
\hline & Paciente 1 (M.C.A.) & Paciente 2 (F.A.C.) & Paciente 3 (A.F.C.) \\
\hline Sexo & F & F & F \\
\hline Idade (anos) & 74 & 76 & 70 \\
\hline Setor & CTI & CTI & CD \\
\hline $\begin{array}{c}\text { Valor de } \\
\text { dímero-D } \\
\text { (ng/mL) }\end{array}$ & 97026 & 37580 & \\
\hline $\begin{array}{c}\text { Diagnóstico } \\
\text { principal }\end{array}$ & $\begin{array}{c}\text { Neoplasia de encéfalo } \\
+ \text { pneumonia viral por }\end{array}$ & pneumonia viral por & DPOC, DRC em \\
& COVID 19 & COVID 19 & último estágio
\end{tabular}

Fonte: Dados de prontuários dos pacientes. 
Disto se verifica que os valores de dímero - D significativamente mais elevados nestes pacientes pode, facilmente, ter sido influenciado pelas comorbidades pré-existentes, bem como pelo avanço da idade, uma vez que todos estes fatores constituem evidente influência na elevação deste analito (Harper et al., 2007; Johnson et al., 2019; Li et al., 2020; Rostami \& Mansouritorghabeh, 2020; Zhang et al., 2020), não se podendo correlacionar, apenas com estes dados, a proeminente elevação dos valores de dímero - D com o diagnóstico de COVID - 19 nos pacientes 1 e 2, reforçando sua inespecificidade e necessidade de correlação com a clínica do paciente.

\section{Conclusão}

No contexto deste Hospital Universitário, não fossem as medidas emergenciais adotadas pela administração no intuito de preservar a continuidade na oferta dos serviços prestados pelo Laboratório de Análises Clínicas, o rápido esgotamento do saldo para aquisição dos reagentes de dímero-D, numa situação de alta exigência deste insumo, como tem sido durante todo o decurso da pandemia, poderia comprometer sobremaneira a garantia da qualidade e gerado agravos imensuráveis aos pacientes deste Hospital, que poderiam não ter suas solicitações atendidas.

Maiores estudos nesse quesito seriam oportunos, a fim de se aprimorar o fluxo resolutivo para situações de calamidade que, infelizmente, possam advir.

Para tanto, uma possibilidade que não deve ser descartada seria a realização de gestões favoráveis a um pronto acionamento dos meios de defesa estatais a fim de viabilizar resposta imediata frente à necessidade urgente do transporte de carga envolvendo, não somente medicamentos, mas outros insumos importantes, tais como reagentes utilizados no auxílio diagnóstico de doenças com alta infectividade.

Embora o dímero-D não tenha sua especificidade elevada enquanto ferramenta de diagnóstico, tem sido muito útil como um preditor de gravidade na pré internação por COVID-19 e seu monitoramento constitui importante auxílio na prática clínica.

\section{Referências}

BRASIL, M. da S. (2020). Protocolo de Manejo Clínico da Covid-19 na Atenção Especializada. In Ministério da Saúde.

Chen, N., Zhou, M., Dong, X., Qu, J., Gong, F., Han, Y., Qiu, Y., Wang, J., Liu, Y., Wei, Y., Xia, J., Yu, T., Zhang, X., \& Zhang, L. (2020). Epidemiological and clinical characteristics of 99 cases of 2019 novel coronavirus pneumonia in Wuhan, China: a descriptive study. The Lancet, 395(10223), 507-513. https://doi.org/10.1016/S0140-6736(20)30211-7

Connors, J. M., \& Levy, J. H. (2020). COVID-19 and its implications for thrombosis and anticoagulation. Blood, 135(23), 2033-2040. https://doi.org/10.1182/BLOOD.2020006000

Cunningham, A. C., Goh, H. P., \& Koh, D. (2020). Treatment of COVID-19: Old tricks for new challenges. In Critical Care (Vol. 24, Issue 1). BioMed Central Ltd. https://doi.org/10.1186/s13054-020-2818-6

Debuc, B., \& Smadja, D. M. (2021). Is COVID-19 a New Hematologic Disease? Stem Cell Reviews and Reports, 17(1), 4-8. https://doi.org/10.1007/s12015020-09987-4

Lúcia, M. da R. S. P. (2005). Universidade Federal do Rio Grande do Sul Faculdade de Medicina Programa de Pós-Graduação em Medicina: Ciências Médicas Associação entre os níveis de D-dímero, produtos de degradação da fibrina/fibrinogênio (PDF) e troponina cardíaca $T$ na investigação dos distúrbios tromboembólicos RAFAEL NOAL MORESCO.

Gabbai-Armelin, P. R., Oliveira, A. B., Ferrisse, T. M., Sales, L. S., Barbosa, E. R. O., Miranda, M. L., Salomão, K. B., \& Brighenti, F. L. (2021). COVID-19 (SARS-CoV-2) infection and thrombotic conditions: A systematic review and meta-analysis. European Journal of Clinical Investigation. https://doi.org/10.1111/eci.13559

Hanff, T. C., Mohareb, A. M., Giri, J., Cohen, J. B., \& Chirinos, J. A. (2020). Thrombosis in COVID-19. In American Journal of Hematology (Vol. 95, Issue 12, pp. 1578-1589). Wiley-Liss Inc. https://doi.org/10.1002/ajh.25982

Harper, P. L., Theakston, E., Ahmed, J., \& Ockelford, P. (2007). D-dimer concentration increases with age reducing the clinical value of the D-dimer assay in the elderly. Internal Medicine Journal, 37(9), 607-613. https://doi.org/10.1111/j.1445-5994.2007.01388.x

Higuera-de-la-Tijera, F., Servín-Caamaño, A., Reyes-Herrera, D., Flores-López, A., Robiou-Vivero, E. J. A., Martínez-Rivera, F., Galindo-Hernández, V., Rosales-Salyano, V. H., Casillas-Suárez, C., Chapa-Azuela, O., Chávez-Morales, A., Jiménez-Bobadilla, B., Hernández-Medel, M. L., Orozco-Zúñiga, B., Zacarías-Ezzat, J. R., Camacho, S., \& Pérez-Hernández, J. L. (2021). The Age-AST-D Dimer (AAD) Regression Model Predicts Severe COVID-19 Disease. 
Research, Society and Development, v. 10, n. 14, e148101421666, 2021

(CC BY 4.0) | ISSN 2525-3409 | DOI: http://dx.doi.org/10.33448/rsd-v10i14.21666

Disease Markers, 2021, 6658270. https://doi.org/10.1155/2021/6658270

Jin, J. M., Bai, P., He, W., Wu, F., Liu, X. F., Han, D. M., Liu, S., \& Yang, J. K. (2020). Gender Differences in Patients With COVID-19: Focus on Severity and Mortality. Frontiers in Public Health, 8. https://doi.org/10.3389/fpubh.2020.00152

Johnson, E. D., Schell, J. C., \& Rodgers, G. M. (2019). The D-dimer assay. American Journal of Hematology, 94(7), 833-839. https://doi.org/10.1002/ajh.25482

Li, Y., Zhao, K., Wei, H., Chen, W., Wang, W., Jia, L., Liu, Q., Zhang, J., Shan, T., Peng, Z., Liu, Y., \& Yan, X. (2020). Dynamic relationship between D-dimer and COVID-19 severity. British Journal of Haematology, 190(1), e24-e27. https://doi.org/10.1111/bjh.16811

Moreno, G., Carbonell, R., Bodí, M., \& Rodríguez, A. (2021). Systematic review of the prognostic utility of D-dimer, disseminated intravascular coagulation, and anticoagulant therapy in COVID-19 critically ill patients. Medicina Intensiva, 45(1), 42-55. https://doi.org/10.1016/j.medin.2020.06.006

Pereira, A., Shitsuka, D., Parreira, F., \& Shitsuka, R. (2018). Método Qualitativo, Quantitativo ou Quali-Quanti. In Metodologia da Pesquisa Científica. https://repositorio.ufsm.br/bitstream/handle/1/15824/Lic_Computacao_Metodologia-Pesquisa-Cientifica.pdf?sequence=1.

Quacken, F., Greiffendorf, I., Ropertz, S., Peters, D., Bergrath, S., Aretz, S., Lahm, A., Pesch, A., Wimmer-Dahmen, M.-L., \& Graeven, U. (2021). [Hospital inhouse contact tracing-significant impact to patient care during the coronavirus pandemic]. Bundesgesundheitsblatt, Gesundheitsforschung, Gesundheitsschutz. https://doi.org/10.1007/s00103-021-03330-z

Rahi, M. S., Jindal, V., Reyes, S. P., Gunasekaran, K., Gupta, R., \& Jaiyesimi, I. (2021). Hematologic disorders associated with COVID-19: a review. Annals of Hematology, 100(2), 309-320. https://doi.org/10.1007/s00277-020-04366-y

Rostami, M., \& Mansouritorghabeh, H. (2020). D-dimer level in COVID-19 infection: a systematic review. In Expert Review of Hematology (Vol. 13, Issue 11, pp. 1265-1275). Taylor and Francis Ltd. https://doi.org/10.1080/17474086.2020.1831383

Velicu, M. A., Furlanetti, L., Jung, J., \& Ashkan, K. (2021). Epidemiological trends in COVID-19 pandemic: Prospective critical appraisal of observations from six countries in Europe and the USA. BMJ Open, 11(4), 1-9. https://doi.org/10.1136/bmjopen-2020-045782

Weitz, J. I., Fredenburgh, J. C., \& Eikelboom, J. W. (2017). A Test in Context: D-Dimer.

WHO. (2010). Relatório Mundial de Saúde - Financiamento dos Sistemas de Saúde. O capminho para a cobertura universal. Relatório Mundial Da Saúde, 1143.

Wu, C., Chen, X., Cai, Y., Xia, J., Zhou, X., Xu, S., Huang, H., Zhang, L., Zhou, X., Du, C., Zhang, Y., Song, J., Wang, S., Chao, Y., Yang, Z., Xu, J., Zhou, X., Chen, D., Xiong, W., \& Song, Y. (2020). Risk Factors Associated with Acute Respiratory Distress Syndrome and Death in Patients with Coronavirus Disease 2019 Pneumonia in Wuhan, China. JAMA Internal Medicine, 180(7), 934-943. https://doi.org/10.1001/jamainternmed.2020.0994

Zhang, L., Yan, X., Fan, Q., Liu, H., Liu, X., Liu, Z., \& Zhang, Z. (2020). D-dimer levels on admission to predict in-hospital mortality in patients with Covid19. Journal of Thrombosis and Haemostasis, 18(6), 1324-1329. https://doi.org/10.1111/jth.14859 